\title{
Comparison of wear characteristics of etched-silicon and carbon nanotube atomic-force microscopy probes
}

\author{
T. Larsen and K. Molonia) \\ PIEZOMAX Technologies, Inc., Middleton, Wisconsin 53562 \\ F. Flack, M. A. Eriksson, and M. G. Lagally \\ University of Wisconsin-Madison, Madison, Wisconsin 53706 \\ C. T. Black \\ IBM T.J. Watson Research Center, Yorktown Heights, New York 10598
}

(Received 19 October 2001; accepted for publication 27 December 2001)

\begin{abstract}
The resolution and wear properties of carbon nanotube and etched-silicon atomic force microscopy probes are compared in intermittent-contact mode. Carbon nanotube probes have at least 20 times the life of etched-silicon probes and provide better resolution at all stages. Sample wear is minimized with carbon nanotube probes. (C) 2002 American Institute of Physics.
\end{abstract}

[DOI: $10.1063 / 1.1452782]$

Since the discovery of carbon nanotubes (CNTs) in $1991{ }^{1}$ much has been done to characterize their properties and explore their potential applications. Although many of these potential uses are still in the nascent stage, it has become clear that CNTs, because of their geometry and unique mechanical properties, are very well suited for scannedprobe microscopy probes, ${ }^{2}$ in particular atomic-force microscopy $(\mathrm{AFM}),{ }^{3}$ but also magnetic-force microscopy ${ }^{4}$ and electric-force microscopy. ${ }^{5}$ CNTs are preferred probes for topographic imaging because they (1) provide improved resolution (uniformly good end form), (2) allow investigating deep and/or narrow surface features (very high aspect ratio and long length), (3) enable probing sensitive and/or easily damaged surface features (mechanical properties of the CNT), (4) have long life (anecdotal evidence of a factor of 10 increased life without degradation of resolution), and (5) provide enhanced capabilities for scanning in water (hydrophobicity of the CNT). ${ }^{6}$

In this letter we quantify the improved resolution, the long life and the superior imaging properties of CNT probes on fragile samples. We compare the wear and degradation of conventional commercial etched-silicon (ES) probes with those of multiwall CNTs during intermittent-contact imaging. We confirm at least an order of magnitude longer life of the CNT without degradation of resolution; in fact we are not able to find a reduction of resolution or any wear of the CNT even after more than two meters of scanning (over 1000 $2 \mu \mathrm{m} \times 2 \mu \mathrm{m}$ images). Samples imaged with CNT probes also show negligible wear compared with those imaged with ES probes.

Figure 1 shows a comparison of the resolution achievable with CNT (a) and ES (b) tips, using $10 \mathrm{~nm}$ Co spheres on $\mathrm{Si}(111)$. The end form of tips made from multiwall CNTs or CNT bundles is invariably better than $20 \mathrm{~nm}$ in diameter, and can be made as small as $3 \mathrm{~nm}$, providing resolution of this order. Although new ES tips can sometimes achieve the resolution of a CNT (we have never observed it to be better

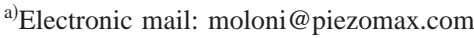

than that of a CNT), their resolution begins to degrade within two or three scans in most cases. Figure 1 also shows a comparison of the ability of the CNT tips to probe deep features (c) versus that of an ES tip (d). Because they are long, narrow tubes, CNTs have a high aspect ratio (tubes with lengths from nanometers to several micrometers can be fabricated as metrology probes). Conventional (ES) probes have a pyramidal shape, which is apparent in the AFM image of a deep trench. Thus, CNT probes can profile morphologies that are inaccessible to ES probes.

The wear characteristics of conventional ES tips were compared with ProbeMAX $\odot$ CNT tips by imaging a polycrystalline silicon (polySi) surface using a Digital Instruments Dimension 3100 AFM in intermittent-contact mode. The CNT probes were prepared by mounting multiwall CNTs onto commercial ES probes and altering the length and end form of the CNT. ${ }^{7,8}$ We used polycrystalline silicon because it is inherently rough, providing a good resolution test, and fairly hard, providing a good test of tip wear. We imaged a $2 \mu \mathrm{m} \times 2 \mu \mathrm{m}$ area at a scan rate of $\sim 2 \mathrm{~Hz}$, with the AFM set to capture images continuously. The set point was kept at high values (low contact forces), throughout scanning. Electron micrographs of the AFM probes before they were used and periodically during the testing were obtained with a JEOL CM200X scanning transmission electron microscopy (STEM). Several ES and CNT tips were evaluated.

Figure 2 demonstrates the results. Figure 2(a) shows the initial AFM scan with an ES probe (all ES probes were initially very similar). Figure 2(b) shows the AFM image obtained during the 35th scan. It is apparent that the resolution is worse already after 35 scans, a total length of scan of only $7 \mathrm{~cm}$. The final image is taken on a different area of the sample to avoid convolution of tip wear and sample wear (if any). The measured rms roughness of the polySi changes from $2.87 \mathrm{~nm}$, for the initial scan, to $2.62 \mathrm{~nm}$, after the 35 th scan, indicating the blunting of the tip. Tip wear is not observed with a CNT probe. Figure 2(c) shows the initial AFM scan of polySi using a CNT tip. The measured rms roughness $(3.14 \mathrm{~nm})$ is almost $20 \%$ higher than that seen with a new ES 


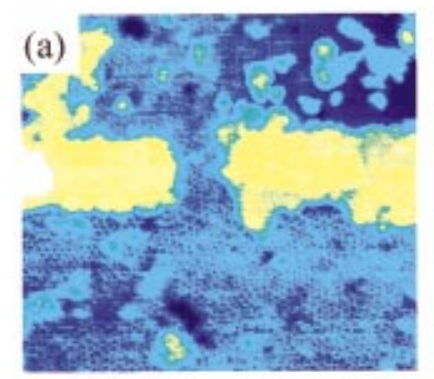

$\overline{200 \mathrm{~nm}}$
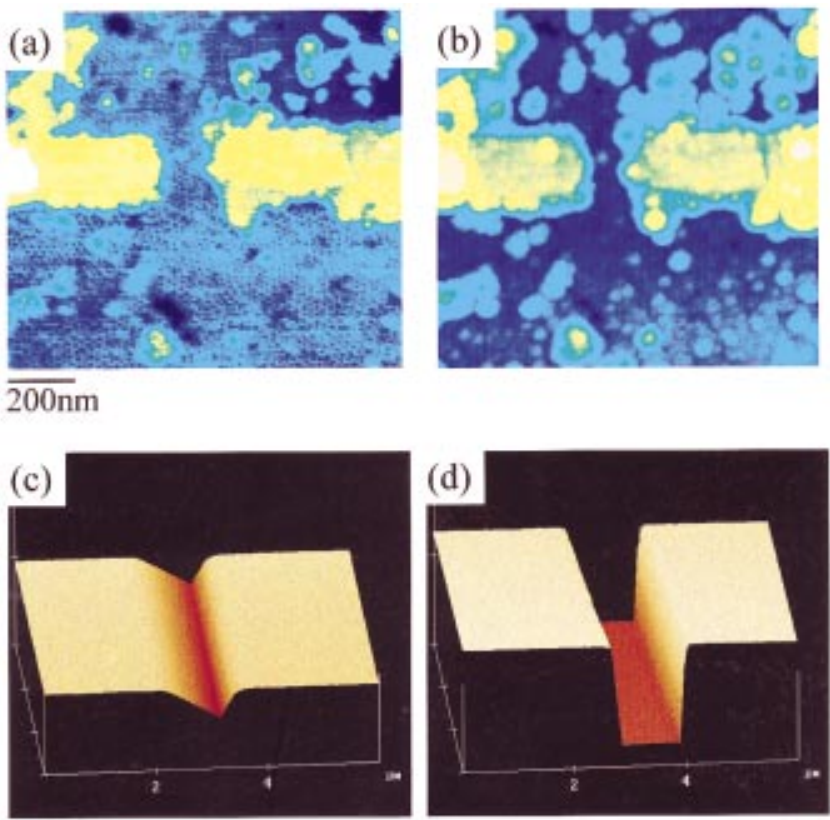

FIG. 1. (Color) AFM images of a monolayer of $10 \mathrm{~nm}$ Co spheres deposited on $\mathrm{Si}(111)$ imaged (a) with a CNT probe, (b) with a new ES tip. Note the resolution of the Co balls evident with the CNT probe. Yellow (higher) regions are deposited $\mathrm{Au}$ contacts. Lighter blue regions are second and third layers of the Co deposit. Comparison of AFM images using (c) ES and (d) CNT probes, of a $2 \mu \mathrm{m}$ deep, $1 \mu \mathrm{m}$ wide trench, illustrating the ability of CNT probes to image very deep features. The image and depth of penetration into the trench with the ES tip reflects the tip shape.
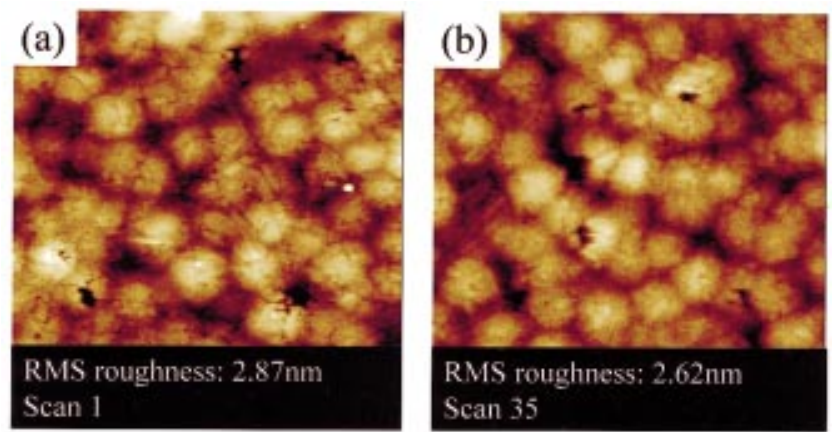

\section{$500 \mathrm{~nm}$}
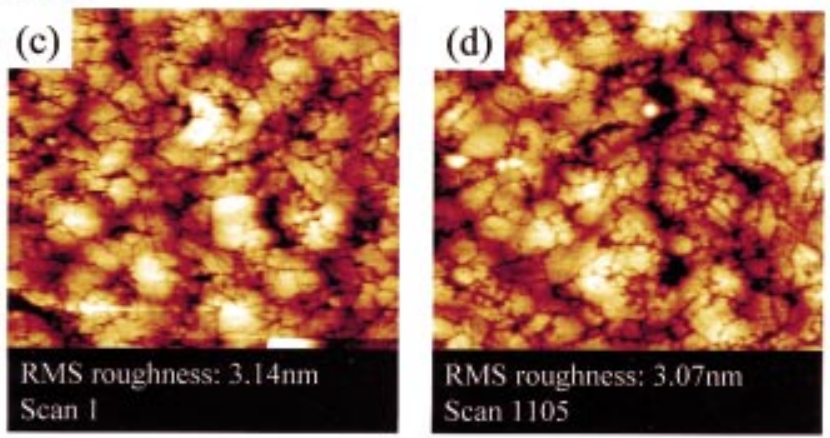

FIG. 2. (Color) Image resolution degradation using a conventional ES probe (a) and (b). The degradation is clearly apparent by the 35th scan: the image appears more blurred. As expected, the measured rms roughness is lower. Image resolution comparison using a CNT probe (c) and (d). No image degradation is observed after more than 1000 scans, as is apparent by the

nearly unchanging measured rms roughness values.
Downloaded 21 Feb 2007 to 128.104.198.71. Redistribution subject to AIP lice
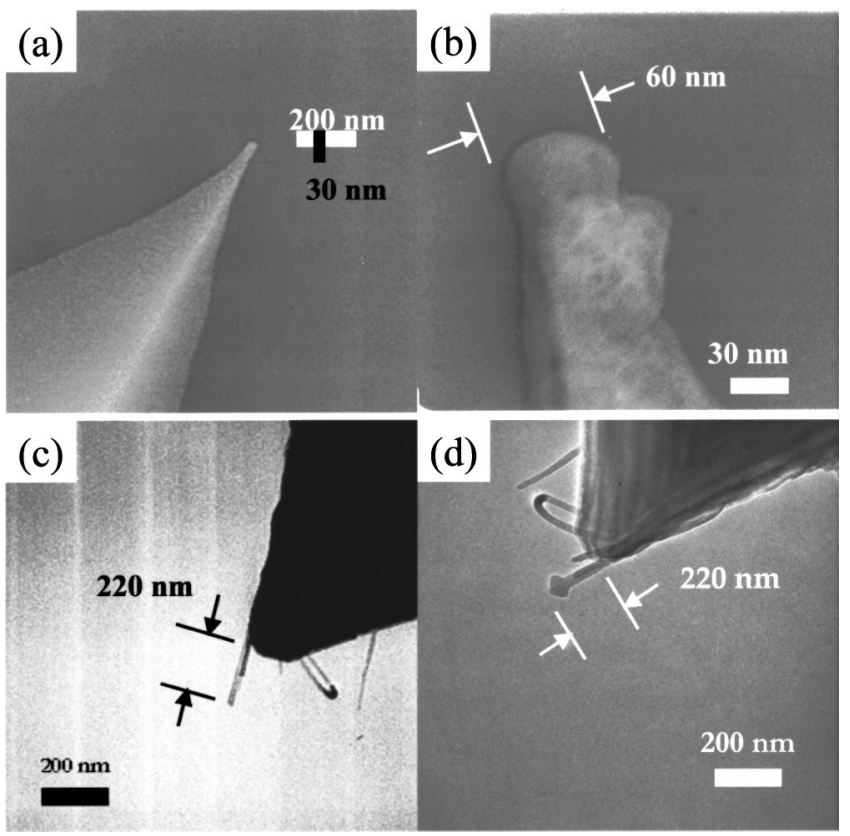

FIG. 3. TEM image of a new ES tip (a) before use and (b) after 180 scans. It has obviously become blunted. This particular probe acquired contamination on its side during use. TEM images of the CNT probe used to acquire the AFM data of Fig. 2, (c) initially, (d) after 1105 scans. The probe has not changed its length. A "ball" of apparent contamination appears away from the end of the tube; the resulting shape may be useful for sidewall probing.

tip, indicating better ability to track surface morphological features because of the higher resolution of the CNTs. We observed no change in resolution for any CNT, in some cases acquiring more than $10002 \mu \mathrm{m} \times 2 \mu \mathrm{m}$ images, corresponding to over two meters of scanning.

Figure 3 shows TEM images of an ES tip initially [Fig. 3(a)] and after it was used for 180 scans [Fig. 3(b)]. It has obviously become blunted. Early in the testing this particular probe also acquired a bit of contamination on its side, which we used to monitor periodically changes in the end form during the 180 scans. We observe a continuous probe degra-

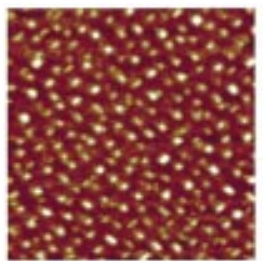
initially

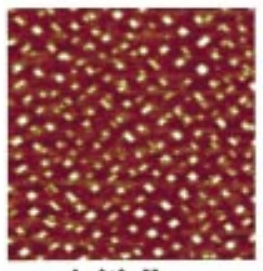

initially

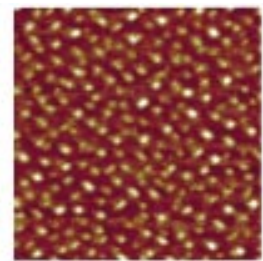
after 1 hour

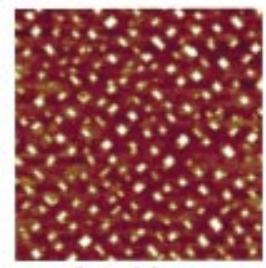

after 1 hour

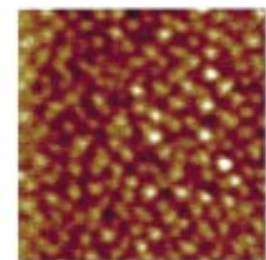

after 15 hours

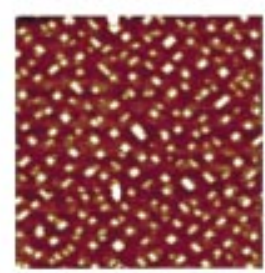

after 9 hours
FIG. 4. (Color) Sample wear by different probes. A comparison of $1 \mu \mathrm{m}$ images over a number of hours repeated on the same spot on a sample containing very small SiGe three-dimensional islands (huts) using ES (top row: initial, after $1 \mathrm{~h}$, after $15 \mathrm{~h}$ ) and $\mathrm{CNT}$ (bottom row: initial, after $1 \mathrm{~h}$, after $9 \mathrm{~h}$ ) probes. Image degradation is noticeable already after $1 \mathrm{~h}$ of imaging with an ES tip, while there is none even after $9 \mathrm{~h}$ of imaging with a CNT tip.

o AlP license or copyright, see http://apl.aip.org/apl/copyright.jsp 
dation. It is clear that the force during intermittent-contact scanning is sufficient to cause wear of the $\mathrm{Si}$ at the tip. We have observed no catastrophic failures of CNT tips during scanning. Figures 3(c) and 3(d) show TEM images of the probes used to acquire the AFM data of Fig. 2. There is neither a shortening of the CNT nor a change in the end form. The decrease in measured rms roughness is within the error of such determinations, changing from 3.14 to $3.07 \mathrm{~nm}$ after more than 1000 scans. We have made many measurements with ES and CNT tips that support the results shown here, though we have not used any other CNT tip for 1100 scans. We observed that CNTs do not easily break and there is anecdotal evidence that they do not wear rapidly. These features are essential for several reasons: (1) no change in resolution over time; (2) reliability-no debris left in any of the regions that have been probed; and (3) reduced down time for probe changes, a significant advantage for a realtime, on-line tool, such as in process inspection and characterization of advanced microelectronic device structures.

In a related experiment we investigated sample damage caused by CNT and ES probes. We imaged SiGe quantum dots grown on $\mathrm{Si}(001)$ using intermittent-contact mode. These quantum dots are regularly shaped pyramids bounded by (105) facets with a typical height of $<5 \mathrm{~nm}$ and base width of $<50 \mathrm{~nm} .{ }^{9}$ Figure 4 shows a comparison of images over a number of hours repeated on the same spot on the sample, using ES (top row) and CNT (bottom row) probes. Again, the initial resolution provided by the CNT tip is better than that by the ES tip. Image degradation is noticeable already after $1 \mathrm{~h}$ of imaging with an ES tip, while there is none even after $9 \mathrm{~h}$ of imaging with a CNT tip. We confirm surface degradation, in the case of the ES tip, by imaging a virgin area after the above scans are completed and observing a better image than on areas that have been repeatedly scanned. We can conclude that with CNT tips, neither the sample nor the probe is affected by the act of imaging. The most reasonable explanation for this combined behavior is that the CNT probe will buckle to absorb the force at contact. It is well known that CNTs are able to buckle without breaking. ${ }^{10}$
ES probes are widely used. Our results reinforce their limitations in terms of rapid resolution degradation with use and the inability to image high-aspect-ratio features. We have shown that CNT tips do not have these limitations. If accurate roughness values to better than $20 \%$ are important, even a new ES tip is not satisfactory. No metric exits for deciding when a tip is "worn out," as this definition must depend on the initial resolution that is required to image the morphology of interest. If we take degradation of the rms roughness value as a measure of resolution and if we take an rms value change of $10 \%$ as the metric of a worn tip, then our results on polySi indicate a useful life of about $10 \mathrm{~cm}$ of scan length for an ES tip. A CNT tip has a useful life of at least 20 times higher, with the upper limit not known because we have not been able to determine this limit. This long life, the lack of debris from the tip, and the ability to probe deep holes and vias should make CNT tips the ideal probe for surface metrology in the semiconductor and related industries. Their gentleness as a probe additionally provides benefits in imaging easily damaged materials.

The work of T.L. and K.M. was supported by DARPA, SBIR Contract No. DAAH01-00-C-R221, awarded to PIEZOMAX Technologies. The work of F.F., M.E., and M.L. was supported by NSF.

${ }^{1}$ S. Iijima, Nature (London) 354, 147 (1991).

${ }^{2}$ H. Dai, J. H. Hafner, A. G. Rinzler, D. T. Colbert, and R. E. Smalley, Nature (London) 384, 147 (1996).

${ }^{3}$ G. Binning, C. F. Quate, and C. Gerber, Phys. Rev. Lett. 56, 930 (1986).

${ }^{4} \mathrm{P}$. Eames and K. Moloni (unpublished).

${ }^{5}$ S. B. Arnason, A. G. Rinzler, Q. Hudspeth, and A. F. Hebard, Appl. Phys. Lett. 75, 2842 (1999).

${ }^{6}$ K. Moloni, M. Buss, and R. Andres, Ultramicroscopy 80, 237 (1999).

${ }^{7}$ U.S. Patent Appl. Serial No. 09/621,710, Katerina Moloni, July 24, 2000.

${ }^{8}$ K. Moloni, A. Lal, and M. G. Lagally, Proc. SPIE 4098, 76 (2000).

${ }^{9}$ C. Teichert, J. Tersoff, M. G. Lagally, in Morphological Organization in Epitaxial Growth and Removal, edited by Z. Zhang and M. G. Lagally (World Scientific, Singapore, 1999).

${ }^{10}$ O. Lourie, D. M. Cox, and H. D. Wagner, Phys. Rev. Lett. 81, 1638 (1998). 